\title{
A k-Nearest Neighbours Classifier for Predicting Catheter Ablation Responses Using Noncontact Electrograms During Persistent Atrial Fibrillation
}

\author{
Xin $\mathrm{Li}^{1}$, Gavin S Chu ${ }^{1,2}$, Tiago P Almeida ${ }^{5}$, João L Salinet ${ }^{6}$, Amar R Mistry ${ }^{1}$, Zakariyya Vali ${ }^{1}$, \\ Peter J Stafford ${ }^{2}$, Fernando S Schlindwein ${ }^{3,4}$, G André $\mathrm{Ng}^{1,2,3}$ \\ ${ }^{1}$ Department of Cardiovascular Sciences, University of Leicester, Leicester, UK \\ ${ }^{2}$ University Hospitals of Leicester NHS Trust, UK \\ ${ }^{3}$ National Institute for Health Research Leicester Cardiovascular Biomedical Research Centre, UK \\ ${ }^{4}$ Department of Engineering, University of Leicester, Leicester, UK \\ ${ }^{5}$ Aeronautics Institute of Technology, ITA, Brazil \\ ${ }^{6}$ Biomedical Engineering, Centre for Engineering, Modelling and Applied Social Sciences, Federal \\ ABC University, Brazil
}

\begin{abstract}
The mechanisms for the initiation and maintenance of atrial fibrillation $(A F)$ are still poorly understood. Identification of atrial sites which are effective ablation targets remains challenging. Supervised machine learning has emerged as an effective tool for handling classification problems with multiple features. The main goal of this work is to use learning algorithms in predicting the responses of ablating electrograms and their effect on terminating $A F$ and the cycle length changes. A total of 3,206 electrograms (EGMs) from ten persistent $A F$ (pers $A F$ ) patients were used. 5-fold cross-validation was applied, in which $80 \%$ of the data were used as training set and 20 $\%$ used as validation. Dominant frequency $(D F)$ and organisation index (OI) were calculated from EGMs (264 seconds) for all patients and used as input features. A $k$-nearest neighbour (KNN) classifier was trained using ablation lesion data and deployed in additional 17,274 EGMs that were not ablated. The classification accuracy of $85.2 \%$ was achieved for the KNN classifier.

We have proposed a supervised learning algorithm using $D F$ features, which has shown the ability of accurately performing EGM signal classification that could be potentially used to identify ablation targets and become a robust real-time patient diagnosis system.
\end{abstract}

\section{Introduction}

Atrial fibrillation (AF) is the commonest cardiac arrhythmia in clinical practice, affecting 1-2\% of the general population [1]. The loss of effective atrial contraction in AF patients may result in blood clots in the atria, which increases the risk of stroke five-fold [2]. Although catheter ablation techniques are widely applied to treat AF patients, the underlying pathophysiological mechanisms of persistent $\mathrm{AF}$ (persAF) remain unclear [3]. Atrial sites hosting high dominant frequency (DF) might be the important sites for the maintenance of AF [4]. DF has shown to be spatiotemporally unstable [5], and temporally stable DF showed the potential to represent relevant and stable focal drivers [6]. Panoramic mapping using electrogram (EGM) recordings with longer duration provides excellent data for assessing frequency features such as DF and organisation index (OI) and their temporal stability and variation. Machine learning techniques have been successfully used in variety of scientific fields, including medicine [7]. The knearest neighbour (KNN) algorithm as one of the simplest among machine learning algorithms, despite its simplicity, can often outperform more powerful classifiers. In this study, we aim to investigate the performance of KNN algorithm in predicting the ablation responses of atrial EGMs.

\section{Materials and Methods}

\subsection{Data Acquisition}

Ten persAF patients undergoing first time left atrial (LA) catheter ablation were enrolled. High DF regions in the LA were identified as described before [5] and 30s of LA non-contact electrograms (EGMs, Ensite Array, St Jude Medical, 2048 channels) were exported to our Matlab platform [8] to guide ablation targeting DF. Four out of ten patients had AF terminations before pulmonary veins isolation (PVI). There were no adverse events in all ten patients. Pre-ablation EGMs up to 5 min duration were exported offline for training and validation for all patients. 


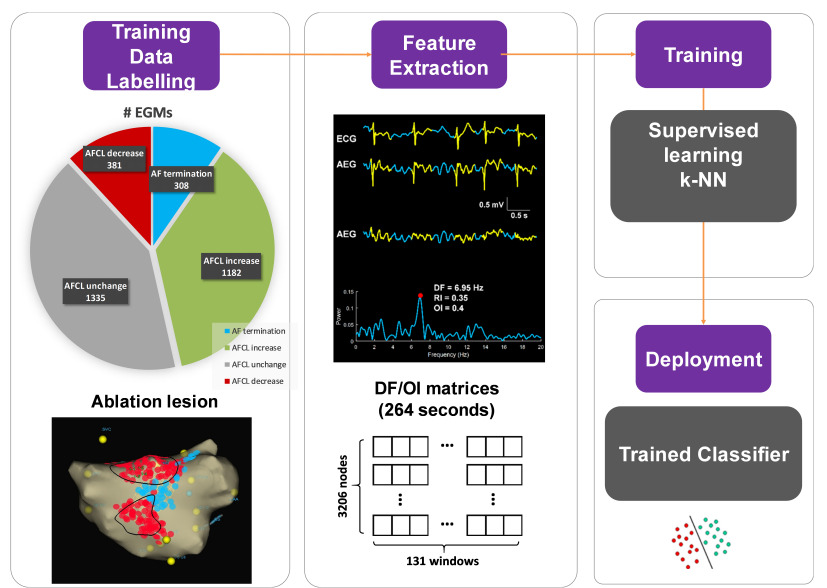

Figure 1. Flow chart of data assembly, processing, and analysis.

\subsection{Training Data Labelling}

AF cycle lengths (AFCL) before and after ablating each atrial DF site (a cluster of lesion points defined by revisiting DF from 30-second data before ablation) were recorded in Labsystem ${ }^{\mathrm{TM}}$ Pro EP Recording System. Four classes of data were considered as labels: 1) AF termination, 2) AFCL increase ( $\geq 10 \mathrm{~ms})$, 3) AFCL unchange and 4) AFCL decrease $(\leq 10 \mathrm{~ms})$. Classes 1 and 2 were considered as positive ablation results. As illustrated in Figure 1 (left panel), a total of 51 atrial locations (3,206 nodes) were ablated: 16 with AFCL increase (1,182 nodes), 4 terminated AF (308 nodes), 7 AFCL decrease (381 nodes) and 24 no AFCL change (1,335 nodes).

\subsection{Signal Processing}

The 5-min EGMs were sampled at 2034.5 Hz and then re-sampled to $512 \mathrm{~Hz}$ to reduce processing time and save storage using cubic interpolation method. Ventricular farfield activity present in the EGMs might appear as misleading frequency components on the atrial frequency spectrum, affecting the accuracy of DF identification, and QRST subtraction was performed as described in our previous work (see middle panel Figure 1) [9].

The EGMs were then divided into 4 seconds windows with a 50\% overlap. For each window, spectral analysis was carried out by performing fast Fourier transform (FFT). A zero padding factor of 5 was applied when performing the FFT, resulting in a frequency step of $0.05 \mathrm{~Hz}$. A Hamming window was used to reduce the amplitude of the side lobes around the DF peak in the power spectrum. DF was defined as the frequency peak in the power spectrum within the physiological range of $4-10 \mathrm{~Hz}$. OI is defined as the ratio of the area of the DF peak $( \pm 0.25 \mathrm{~Hz})$ together with its harmonics, and the total area of the power spectrum (up to $20 \mathrm{~Hz}$ ).

\subsection{Feature Extraction}

The shortest recording duration among the ten patients was used, which is 264 seconds, resulting in 131 windows (4-second long). Four features were extracted for each long EGM segment: average DF, average OI, standard deviation of DF (std DF) and standard deviation of OI (std $\mathrm{OI})$. A feature matrix of $4 \times 3206$ was generated to use as input/predictor of the training model. A categorical matrix of labels $(1 \times 3206)$ was used as responses.

\subsection{Classification Model Training}

Training and classification was implemented in Matlab environment. 5-fold cross-validation was applied as only a total of 51 atrial locations $(3,206$ nodes) were available for training and validation. A KNN classifier was trained on $80 \%$ of data as a train set, and the accuracy was evaluated on $20 \%$ of data as a test set. In KNN classification, an object is usually classified by a majority vote of its neighbours, where the object is consequently assigned to the class that is most common among its KNN. Factors such as $\mathrm{k}$ value, distance calculation and choice of appropriate predictors all have significant impact on the model performance. We have tested several training configurations and the one with highest classification accuracy was selected, where $\mathrm{k}$ is configured as 10 with the square-inversed Euclidean as distance measurements.

\subsection{Model Deployment}

After the model was trained using ablation lesion data, predictions were also made on the EGMs that were not ablated $(17,274)$ for all patients.

\subsection{Statistical Analysis}

All continuous normally distributed variables are expressed as mean \pm SD. Unpaired t test with Welch's correction and one-way ANOVA were applied among each two classes and four classes for all features. P-values of less than 0.05 were considered statistically significant.

To generate receiver operating characteristic (ROC) curve for multi-class classification, it is necessary to transform the output to binary. One-vs-rest (OvR) approach was used and generated four ROC curves and their corresponding area under the curve (AUCs) for each class, considering all three other classes as negative label. 


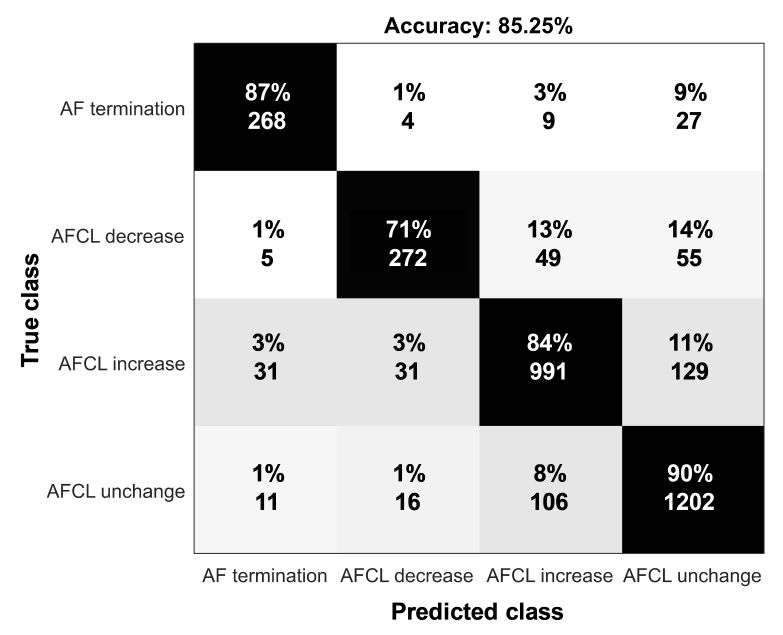

Figure 2. The confusion matrix of the trained KNN classifier. (boxed number and percentage of EGMs predicted for each class)

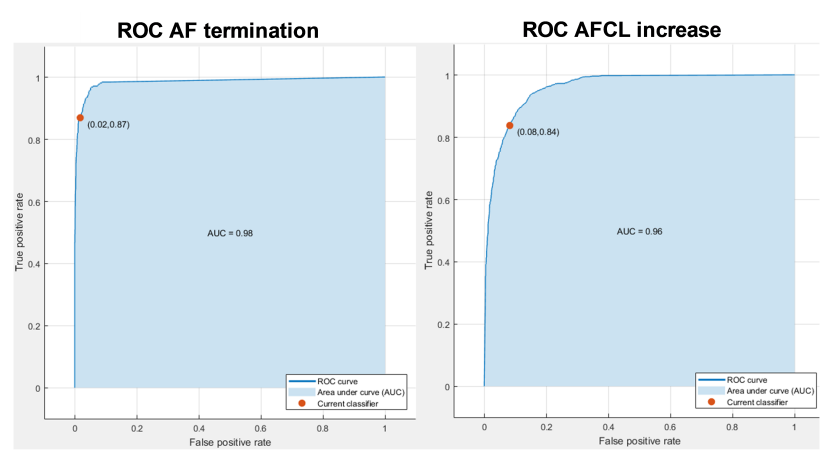

Figure 3. The Receiver operating characteristic Curves of the positive classes.

\section{Results and Discussions}

A total of 3,206 EGMs from ten persAF patients were used, 2,565 were used as training set and 641 used as validation. Four-class KNN classifier was trained with the classification accuracy of $85.2 \%$ achieved.

\subsection{ROC Analysis}

In Figure 2, the confusion matrix of the KNN classier shows the number of EGMs and the true positive rate of each class comparing to the true class. Figure 3 illustrates the ROC curves of the positive classes: $\mathrm{AF}$ termination class (AUC $=0.98$ ) and AFCL increase class (AUC $=0.96$ ). For $\mathrm{AF}$ termination classification from all four classes, a sensitivity of $87 \%$ and a specificity of $98 \%$ were achieved, whilst classifying AFCL increase group resulted in a sensitivity of $84 \%$ and a specificity of $92 \%$.

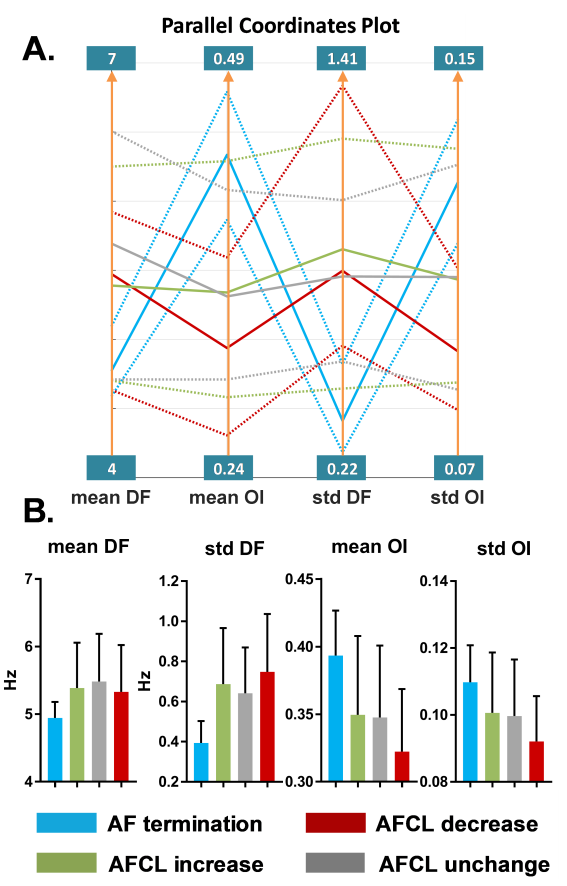

Figure 4. A. The parallel coordinates plot of 4 features for each class; B. The bar graphs of the 4 features for each class.

Table 1. Table of $p$ values of $t$ tests between classes (T: AF termination; $\uparrow:$ AFCL increase; $\sim$ : AFCL unchange; $\downarrow$ : AFCL decrease; ns: not significant).

\begin{tabular}{c|c|c|c|c}
\hline & mean DF & std DF & mean OI & std OI \\
\hline $\mathrm{T}$ vs $\uparrow$ & $<0.0001$ & $<0.0001$ & $<0.0001$ & $<0.0001$ \\
\hline $\mathrm{T}$ vs $\sim$ & $<0.0001$ & $<0.0001$ & $<0.0001$ & $<0.0001$ \\
\hline $\mathrm{T}$ vs $\downarrow$ & $<0.0001$ & $<0.0001$ & $<0.0001$ & $<0.0001$ \\
\hline$\uparrow$ vs $\sim$ & $<0.001$ & $<0.0001$ & $\mathrm{~ns}$ & $\mathrm{~ns}$ \\
\hline$\uparrow \mathrm{vs} \downarrow$ & $\mathrm{ns}$ & $<0.001$ & $<0.0001$ & $<0.0001$ \\
\hline$\sim \mathrm{vs} \downarrow$ & $<0.001$ & $<0.0001$ & $<0.0001$ & $<0.0001$ \\
\hline
\end{tabular}

\subsection{Feature Analysis}

Figure 4 A shows the parallel coordinates plot illustrating the multi-dimension data consisting of 4 features for each class: mean DF and OI, standard deviation of DF and OI (full line is median, dotted lines are the 25th and 75th percentile). The vertical axis is normalised per index, and minimum and maximum values are shown on top and bottom. In Figure 4 B, the bar graphs summarise the mean and standard deviations of all features for each class and the $p$ values between each two classes were calculated (Table 1). The mean DFs of the four classes were $4.94 \pm 0.23,5.38$ $\pm 0.67,5.48 \pm 0.71$ and $5.33 \pm 0.69(\mathrm{p}<0.0001)$. The temporal DF standard deviations were $0.39 \pm 0.11,0.69 \pm$ $0.28,0.64 \pm 0.23$ and $0.75 \pm 0.29(\mathrm{p}<0.0001)$. The mean OIs of the four classes were $0.39 \pm 0.3,0.35 \pm 0.06,0.35$ \pm 0.05 and $0.32 \pm 0.05(\mathrm{p}<0.0001)$. The temporal OI 


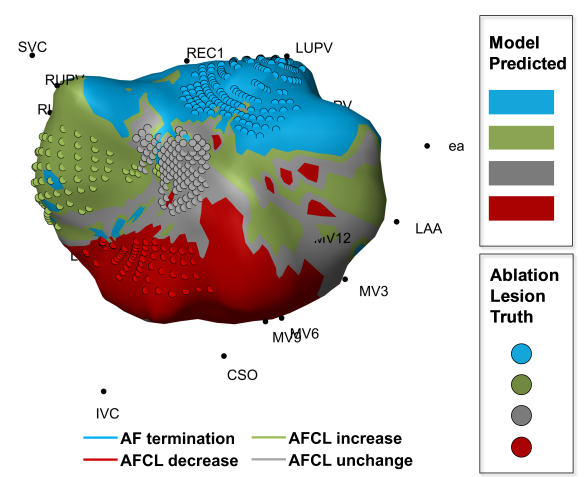

Figure 5. 3D LA colour-coded by classification labels.

standard deviations were $0.11 \pm 0.01,0.10 \pm 0.02,0.10 \pm$ 0.02 and $0.09 \pm 0.01(\mathrm{p}<0.0001)$. It is notable that most features in the AF termination class were significantly different from all other classes, indicating that atrial regions that are responsible for AF termination did show distinct characteristics, and termination data from more patients may improve the classification dealing with future data.

\subsection{Classification Model on New Data}

Figure 5 is a 3D example of one patient with the classification model applied on all data points (2048) including the nodes/EGMs that were not ablated, to predict the response of ablation measured by AFCL changes and classified into four classes. The LA is colour-coded by the prediction of using the trained KNN classifier whilst the colour-coded dots are the ablation lesions with the actual AFCL responses. It is expected that a larger atrial region would be labelled as each class, but the accuracy of the unablated regions can not be verified. More ablation data from more patients would help to further refine the classifier and enrich the knowledge of the classifier,especially on the inter-patient differences.

\section{Conclusions}

This paper presents a framework to identify EGMs that are responsible for maintenance of persAF and potential targets for catheter ablation using panoramic non-contact mapping. Features based on DF and OI were extracted and used to train a KNN classifier to classify the EGMs into AF termination, AFCL increase, AFCL unchange and AFCL decrease. AF termination class showed lower signal frequency, higher temporal stability, higher organisation and lower organisation stability. These important findings may suggest that targeting atrial regions with appropriate frequency characteristics might improve ablation outcome in persAF. The promising results of this study warrant future study with a larger data set and real time implementation of software tool to guide ablation. The proposed algorithm using shorter signal length should be evaluated in future studies.

\section{Acknowledgements}

This work was supported by the NIHR Leicester Biomedical Research Centre. XL received research grants from Medical Research Council, UK. TPA received research grants from Fundação de Amparo à Pesquisa do Estado de São Paulo (FAPESP, n. 2017/00319-8).

\section{References}

[1] Lip GY, Fauchier L, Freedman SB, Van Gelder I, Natale A, Gianni C, Nattel S, Potpara T, Rienstra M, Tse HF, Lane DA. Atrial fibrillation. Nat Rev Dis Primers 2016;2:16016.

[2] Oral H. Atrial Fibrillation: Mechanisms , Features , and Management Pathophysiology, volume Vol. 2000. 5th ed. edition. Philadelphia, Pa.: Saunders, 2009; 119125.

[3] Nattel S. New ideas about atrial fibrillation 50 years on. Nature 2002;415(6868):219-26.

[4] Sanders P, Berenfeld O, Hocini M, Jais P, Vaidyanathan R, Hsu LF, Garrigue S, Takahashi Y, Rotter M, Sacher F, Scavee C, Ploutz-Snyder R, Jalife J, Haissaguerre M. Spectral analysis identifies sites of high-frequency activity maintaining atrial fibrillation in humans. Circulation 2005;112(6):78997.

[5] Salinet JL, Tuan JH, Sandilands AJ, Stafford PJ, Schlindwein FS, Ng GA. Distinctive patterns of dominant frequency trajectory behavior in drug-refractory persistent atrial fibrillation: preliminary characterization of spatiotemporal instability. J Cardiovasc Electrophysiol 2014;25(4):371-379.

[6] Kimata A, Yokoyama Y, Aita S, Nakamura H, Higuchi K, Tanaka Y, Nogami A, Hirao K, Aonuma K. Temporally stable frequency mapping using continuous wavelet transform analysis in patients with persistent atrial fibrillation. J Cardiovasc Electrophysiol 2018;19.

[7] Deo RC. Machine learning in medicine. Circulation 2015; 132(20):1920-1930. ISSN 0009-7322.

[8] Li X, Salinet JL, Almeida TP, Vanheusden FJ, Chu GS, $\mathrm{Ng}$ GA, Schlindwein FS. An interactive platform to guide catheter ablation in human persistent atrial fibrillation using dominant frequency, organization and phase mapping. Comput Methods Programs Biomed 2017;141:83-92.

[9] Salinet J. L. J, Madeiro JP, Cortez PC, Stafford PJ, Ng GA, Schlindwein FS. Analysis of QRS-T subtraction in unipolar atrial fibrillation electrograms. Med Biol Eng Comput 2013; 51(12):1381-91.

Address for correspondence:

Dr. Xin Li

Department of Cardiovascular Sciences

University of Leicester, UK

x1251@1e.ac.uk 